\title{
New insights into the mechanisms of diabetic complications: role of lipids and lipid metabolism
}

\author{
Stephanie Eid ${ }^{1}$ - Kelli M. Sas ${ }^{2}$ - Steven F. Abcouwer ${ }^{3}$ - Eva L. Feldman ${ }^{1} \cdot$ Thomas W. Gardner $^{3,4}$. \\ Subramaniam Pennathur ${ }^{2,4}$. Patrice E. Fort ${ }^{3,4}$
}

Received: 6 December 2018 / Accepted: 21 May 2019 / Published online: 25 July 2019

(C) Springer-Verlag GmbH Germany, part of Springer Nature 2019

\begin{abstract}
Diabetes adversely affects multiple organs, including the kidney, eye and nerve, leading to diabetic kidney disease, diabetic retinopathy and diabetic neuropathy, respectively. In both type 1 and type 2 diabetes, tissue damage is organ specific and is secondary to a combination of multiple metabolic insults. Hyperglycaemia, dyslipidaemia and hypertension combine with the duration and type of diabetes to define the distinct pathophysiology underlying diabetic kidney disease, diabetic retinopathy and diabetic neuropathy. Only recently have the commonalities and differences in the metabolic basis of these tissue-specific complications, particularly those involving local and systemic lipids, been systematically examined. This review focuses on recent progress made using preclinical models and human-based approaches towards understanding how bioenergetics and metabolomic profiles contribute to diabetic kidney disease, diabetic retinopathy and diabetic neuropathy. This new understanding of the biology of complication-prone tissues highlights the need for organ-specific interventions in the treatment of diabetic complications.
\end{abstract}

Keywords Diabetes $\cdot$ Diabetic complications $\cdot$ Lipid metabolism $\cdot$ Omics $\cdot$ Review $\cdot$ Specific mechanisms

\section{Abbreviations \\ ACCORD Action to Control Cardiovascular Risk in Diabetes \\ FIELD Fenofibrate Intervention and Event Lowering in Diabetes \\ LXR Liver X receptor \\ PPAR Peroxisome proliferator-activated receptor \\ PUFA Polyunsaturated fatty acid}

Electronic supplementary material The online version of this article (https://doi.org/10.1007/s00125-019-4959-1) contains a slideset of the figures for download, which is available to authorised users.

Patrice E. Fort

patricef@med.umich.edu

1 Department of Neurology, University of Michigan, Ann Arbor, MI, USA

2 Division of Nephrology, Department of Internal Medicine, University of Michigan, Ann Arbor, MI, USA

3 Department of Ophthalmology and Visual Sciences, University of Michigan Kellogg Eye Center, 1000 Wall Street, Ann Arbor, MI 48105, USA

4 Department of Molecular and Integrative Physiology, University of Michigan, Ann Arbor, MI, USA

\section{Introduction}

Diabetes continues to be a major public health problem, currently affecting over 425 million people worldwide [1]. This number is expected to increase due to the growing rates of obesity, which contributes to a rising incidence of type 2 diabetes and a projected increase in the prevalence of type 1 diabetes globally $[2,3]$. Despite the different aetiologies of type 1 and type 2 diabetes, both are associated with a number of complications affecting the cardiovascular system, kidneys, eyes and nerves [4]. Cardiovascular disorders, heart failure, atherosclerosis and cerebrovascular events primarily result from damage to the macrovasculature. The other major complications have been long considered as 'microvascular' injuries and manifest as a 'diabetic triopathy' of diabetic kidney disease, diabetic retinopathy and diabetic neuropathy [4]. These complications account for the majority of morbidity and mortality risks associated with diabetes. While glucose and glucose metabolism have been the focus of research to understand the pathophysiology of these complications for decades, altered lipid metabolism has recently received increasing attention as a key player in disease pathology. In this review, we address the recent progress made using preclinical 
models and human-based approaches to shed new light on the relative roles of glucose and lipid metabolism in the onset and progression of diabetic microvascular complications.

\section{Clinical overview of diabetic complications}

Diabetic kidney disease Diabetic kidney disease affects 30 $40 \%$ of all individuals with diabetes and is the leading cause of end-stage renal disease in the USA, accounting for significant morbidity and mortality risks in individuals with type 1 and type 2 diabetes [5]. Clinically, diabetic kidney disease is characterised by increased urinary albumin output and a decline in the glomerular filtration rate, both reflecting a progressive deterioration of renal function [6]. This loss of function coincides with histopathological changes, including glomerular and tubular hypertrophy with gradual thickening of the glomerular and tubular basement membranes, podocyte effacement and eventually glomerulosclerosis and tubulointerstitial fibrosis [7].

Diabetic retinopathy Diabetic retinopathy is the primary cause of blindness among the working-age population worldwide. After 20 years of diabetes, virtually all individuals with type 1 diabetes and more than $60 \%$ of those with type 2 diabetes eventually develop signs of diabetic retinopathy [8]. After years of clinically silent intraretinal changes, vascular tortuosity, retinal haemorrhage, microaneurysm, cotton-wool spots and lipid exudates become evident as features of nonproliferative diabetic retinopathy [9]. Some affected individuals develop pathological retinal neovascularisation, recognised as proliferative diabetic retinopathy. Fluid accumulation within the central neural retina, referred to as diabetic macula oedema (DME), manifests as abnormal retinal thickening and cystoid formation and is the most common cause of visual loss in individuals with diabetic retinopathy $[10,11]$.

Diabetic neuropathy Diabetic neuropathy is a highly prevalent complication of both type 1 and type 2 diabetes, affecting at least $50 \%$ of individuals with diabetes [12]. Various types of peripheral nerve disorders can develop, the most common being distal symmetric polyneuropathy, affecting nerves of the extremities in a bilateral, symmetric pattern and progressing in a distal-to-proximal manner. Distal symmetric polyneuropathy, referred to as diabetic neuropathy from this point onwards, is a highly debilitating complication associated with an increased susceptibility to ulcerations and infections and may eventually lead to lower-limb amputations. In addition to the well-characterised axonal injury [13], diabetes also targets Schwann cells and the vascular endothelium as demonstrated by segmental demyelination and endoneurial microangiopathy [14].

\section{Mechanisms of onset and progression of diabetic complications}

The importance of intensive glycaemic control in reducing the progression of complications in type 1 diabetes was confirmed in the Diabetes Control and Complications Trial (DCCT) and Epidemiology of Diabetes Interventions and Complications (EDIC) trials [15]. Based on these studies, patient care has been focused on intensifying glucose control, which improved but did not prevent the development and progression of all three diabetic complications in type 1 diabetes. In type 2 diabetes, intensive glycaemic control has a modest effect on these complications [16]. The UK Prospective Diabetes Study [17] and the Steno-2 trial [18] demonstrated amelioration of diabetic kidney disease and diabetic retinopathy following 10 years of intensive glucose-lowering therapy in individuals newly diagnosed with type 2 diabetes but no effect on diabetic neuropathy was found. Other large intervention trials failed to report clear beneficial effects of near normalisation of blood glucose levels on all three complications associated with type 2 diabetes. The Action to Control Cardiovascular Risk in Diabetes (ACCORD), Action in Diabetes and Vascular Disease (ADVANCE) and Veteran Affairs Diabetes Trial (VADT) [19] trials showed modest improvements in the early manifestations of diabetic kidney disease, while no improvements in diabetic retinopathy or diabetic neuropathy were observed between the intensive and the conventional therapy groups [20, 21]. Collectively, these studies suggest that glucose alone is not responsible for diabetic complications, especially in individuals with type 2 diabetes. Rather, responsibility lies with a cluster of factors, including obesity, hypertension, dyslipidaemia, inflammation and insulin resistance, which have an impact on the adipose tissue fatty acid metabolism that underlies the onset and progression of diabetic kidney disease, retinopathy and neuropathy [22]. Thus, this review will focus on our increased understanding of the basic physiology underlying the global and tissue-specific changes in cellular metabolism that contribute to the pathogenesis of diabetic complications.

Role of hyperglycaemia in tissue-specific injury The mechanisms underlying hyperglycaemia-mediated cellular damage include formation of advanced glycation end-products, increased oxidative stress, mitochondrial dysfunction and activation of the polyol and hexosamine pathways [23]. While preclinical studies have supported these hypotheses, clinical trials that targeted each of these pathways failed to confirm a direct pathogenic role for hyperglycaemia-induced metabolic imbalance in diabetic individuals $[24,25]$. The disappointing outcomes of these clinical interventions led our group to ask a fundamental question: do complication-prone tissues (i.e. kidney, eye and nerve) metabolise glucose in a similar or tissuespecific manner under diabetic conditions? We combined 
metabolomics with transcriptomics to examine changes in glucose metabolism in the kidney, eye and nerve of a murine model of type 2 diabetes, the leptin receptor knockout BKS $d b / d b$ mouse model. We discovered that glycolytic genes were uniformly upregulated in the kidney and peripheral nerve, while glycolytic metabolites were increased in the kidney and retina but decreased in the peripheral nerve [26]. Furthermore, we systemically administered substrates (glucose, pyruvate or palmitate) labelled with the stable isotope ${ }^{13} \mathrm{C}$ to investigate how the metabolism of each of these substrates differed in the kidney, eye and nerve between diabetic and control mice. Analysis of metabolic flux, or tracing of each substrate through downstream metabolites in glycolysis, the pentose phosphate pathway, $\beta$-oxidation (acylcarnitines) and the tricarboxylic acid (TCA) cycle, identified tissuespecific differences in substrate utilisation in this type 2 diabetic mouse model. Particularly, glucose flux increased in the kidney, moderately increased in the retina and remained unchanged in the nerve (Table 1), highlighting differences in glucose metabolism in diabetes complications [26]. To our knowledge, no other study has examined alterations in glucose metabolism across complications within the same animal model of diabetes. Besides this study, transcriptomic studies,

Table 1 Pathway summary of in vivo metabolic flux analysis in kidney cortex, sciatic nerve and retina from 24 -week-old diabetic mice compared with control mice

\begin{tabular}{|c|c|c|c|c|}
\hline $\begin{array}{l}\text { Isotope-labelled } \\
\text { substrate }\end{array}$ & Glycolysis & $\begin{array}{l}\text { Pentose } \\
\text { phosphate } \\
\text { pathway }\end{array}$ & Acylcarnitines & $\begin{array}{l}\text { TCA } \\
\text { cycle }\end{array}$ \\
\hline \multicolumn{5}{|l|}{$\left[{ }^{13} \mathrm{C}_{6}\right]$ glucose } \\
\hline Kidney & $\uparrow \uparrow$ & $\downarrow$ & & $\uparrow \uparrow$ \\
\hline Nerve & $=$ & ND & & $\downarrow$ \\
\hline Retina & $\uparrow$ & $=$ & & $\uparrow$ \\
\hline \multicolumn{5}{|c|}{$2,3-\left[{ }^{13} \mathrm{C}_{2}\right]-\mathrm{Na}$ pyruvate } \\
\hline Kidney & & & & $\uparrow \uparrow$ \\
\hline Nerve & & & & $\uparrow \uparrow$ \\
\hline Retina & & & & $\uparrow \uparrow$ \\
\hline \multicolumn{5}{|l|}{$\left[{ }^{13} \mathrm{C}_{16}\right]$ palmitate } \\
\hline Kidney & & & $\uparrow$ & $\uparrow \uparrow$ \\
\hline Nerve & & & $\uparrow \uparrow$ & $\uparrow \downarrow$ \\
\hline Retina & & & $\uparrow$ & $\uparrow \downarrow$ \\
\hline
\end{tabular}

Arrows denote change in flux throughout all $(\uparrow \uparrow)$ or part $(\uparrow)$ of a pathway following systemic administration of isotope-labelled substrates. Pathways may also be unchanged (=) or exhibit a mixed response $(\uparrow \downarrow)$, in which substrate-derived label incorporation was increased and decreased into individual metabolites in a pathway. ND indicates that a signal was not detected above background noise. Empty cells correspond to pathways not applicable for the substrate used

Table adapted with permission of the American Society for Clinical Investigation, from [26]; permission conveyed through Copyright Clearance Center, Inc.

TCA, tricarboxylic acid in combination with metabolomic and proteomic analysis of tissues from both animal models of diabetes and human biopsies from diabetic individuals, have implicated both glucosedependent and independent mechanisms [26-33]. Using metabolomics analysis, Priyadarsini et al found no effect of glucose-derived metabolite flux in human corneal stroma of individuals with type 1 and type 2 diabetes [33]. On the other hand, Freeman et al showed an increase in glucose and polyol pathway intermediates in sciatic nerves of a streptozotocin (STZ)-induced rat model of type 1 diabetes using an integrated proteomics and metabolomics approach [32]. Importantly, all these studies support the involvement of key factors other than glucose in the pathogenesis of diabetic kidney disease, retinopathy and neuropathy, including a central role for disturbances in lipid metabolism [26-34].

In the following sections, we will discuss these alternative mechanisms and highlight the utility of systems biology approaches for improving our understanding of the differential roles of glucose and lipid metabolism in tissue-specific complications pathophysiology.

\section{Rise of the role of lipid metabolism in diabetic complications}

The fact that the risk of diabetic complications persists despite improved glycaemic control in type 2 diabetes suggests that other components of the metabolic syndrome may play a role in the onset and progression of these complications. Our group has focused on understanding how dyslipidaemia and tissuespecific lipid metabolism contribute to diabetic kidney disease, retinopathy and neuropathy, and the intersection of lipid metabolism with hyperglycaemia [26] (Fig. 1).

Lipogenesis and dyslipidaemia Dyslipidaemia is defined as elevated levels of plasma triacylglycerols and cholesteryl esters, which are components of low-density lipoprotein (LDL)cholesterol and high-density lipoprotein (HDL)-cholesterol. Increased hepatic lipogenesis and adipose tissue fatty acid metabolism in response to elevated blood glucose and insulin resistance contribute to dyslipidaemia in both type 1 and type 2 diabetes [35, 36]. Multiple preclinical and clinical studies have proposed dyslipidaemia as a major contributor to the pathophysiology of diabetic kidney disease (reviewed in [37]). In the Fenofibrate Intervention and Event Lowering in Diabetes (FIELD) study, treatment with fenofibrate, a lipidlowering drug, was found to reduce albuminuria and delay the decline of renal function in individuals with type 2 diabetes [38]. However, the effect of statins (antihyperlipidemic drugs) on diabetic kidney disease progression remains controversial. While several preclinical and clinical studies have shown a beneficial effect of statin treatment on kidney function [39, 40], other clinical trials failed to show any improvement in estimated glomerular filtration rate in individuals with type 2 diabetes [41, 42]. Additionally, it remains unclear whether the beneficial effects of statin therapy are the result of a 


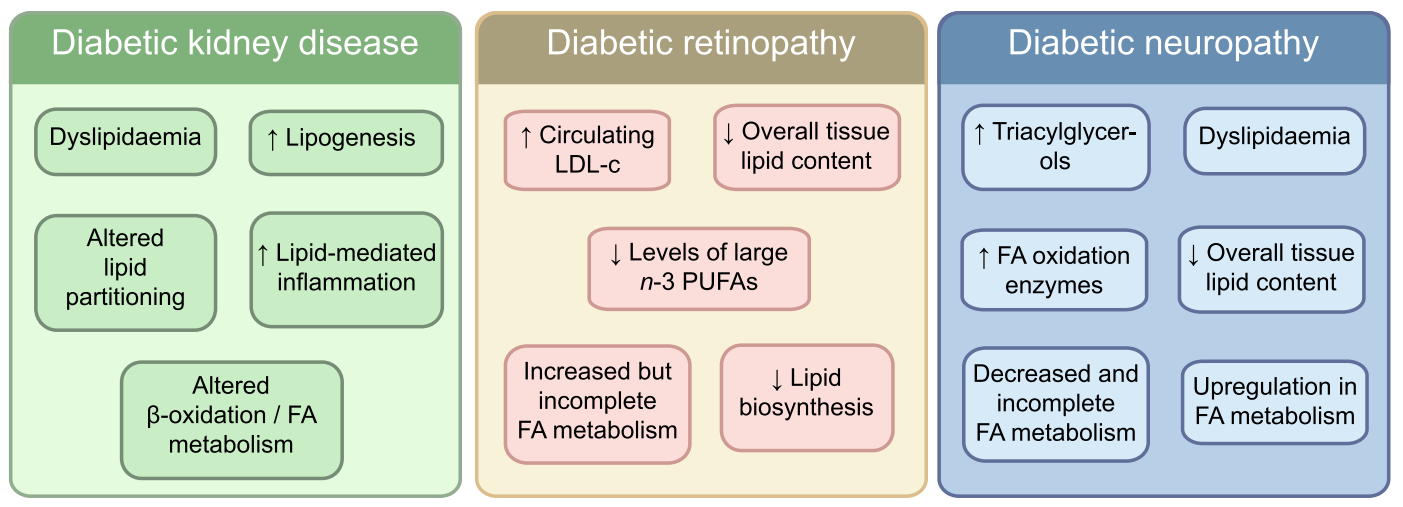

Fig. 1 Systemic and tissue-specific alterations in lipid metabolism specifically associated with the microvascular complications: diabetic kidney disease, diabetic retinopathy and diabetic neuropathy. FA, fatty acid; LDL-c, LDL-cholesterol. This figure is available as part of a downloadable slideset

pleiotropic action of the drug on renal function, independent of its lipid-lowering effect [43].

Controversy also exists regarding the exact role of dyslipidaemia in diabetic retinopathy [44-46]. Several small uncontrolled studies examining the effects of statin use on diabetic retinopathy risk found a beneficial effect [47, 48] but were contradicted by larger studies $[49,50]$. On the other hand, a recent well-conducted large study in Taiwan, of more than 18,000 individuals with type 2 diabetes with age and sexmatched controls, found that statin use significantly reduced retinopathy risk, diminished the incidence of macular oedema and reduced the need for further interventions [51]. Moreover, the FIELD and ACCORD Eye studies found that fenofibrate use significantly lowered the risk of diabetic retinopathy progression and/or the need for laser intervention in diabetic individuals $[52,53]$. However, meta-analysis of studies examining the contribution of serum lipids to the risk of diabetic retinopathy confirmed that while fenofibrate treatment is beneficial, only a slight increase in systemic LDL-cholesterol was associated with diabetic retinopathy risk [54]. Recent studies in diabetic rodents suggest that fenofibrate improves retinal pathology by exerting direct effects on the tissue itself as it improves neuronal survival and function, promotes neuroprotective mechanisms and reduces local inflammation [55-58].

Emerging evidence indicates that dyslipidaemia is a major player in the pathogenesis of diabetic neuropathy $[59,60]$. In these studies, increased plasma triacylglycerols correlated with neuropathy progression in type 2 diabetes while obesity and systemic dyslipidaemia were independently associated with an increased risk of developing neuropathy. In the FIELD study, lipid-lowering therapy was associated with a reduced risk of lower-limb amputations, suggesting that lipid lowering may have beneficial effects on diabetic neuropathy [61]. In the aforementioned study of more than 18,000 individuals with type 2 diabetes from Taiwan, statin therapy significantly reduced the risk of new-onset diabetic neuropathy and foot ulcers [51]. While the mechanisms involved are not fully understood, oxidised LDL-cholesterol induced oxidative stress has been shown to mediate nerve damage in a mouse model of dyslipidaemia-induced neuropathy [62] and likely plays a similar role in neural injury in diabetic neuropathy [13].

Collectively, these results suggest that circulating lipids may have distinct effects on 'microvascular' complications. While increasing evidence suggests an important role for lipid metabolism in diabetic complications, future mechanistic studies are needed to provide more insight into the contribution of dyslipidaemia to diabetes-induced kidney, eye and nerve damage.

\section{Distinct patterns of lipid metabolism in diabetes complica-} tions Studies examining the role of dyslipidaemia in the onset or progression of diabetic complications typically measure plasma levels of LDL, HDL and total triacylglycerols and cholesterol. However, a limitation of measuring the levels of these lipids alone is that this does not effectively reflect the aberrant tissue-specific lipid metabolism associated with diabetes. The complex relationships between plasma lipid levels and diabetic kidney disease, retinopathy and neuropathy suggest that the gross level of these lipid levels in plasma may be inadequate as a biomarker for the prediction of risk of progression. Moreover, glucose is a single molecule but lipids represent a complex mixture of molecular species and, while numerous studies have examined the effects of diabetes on glucose metabolism, few studies have explored how diabetes alters lipid content and metabolism within complicationprone tissues. To address this knowledge gap, our group has used integrated approaches to link systemic and tissue-specific lipid metabolism to diabetic complications pathologies [26, 31]. We have measured levels of multiple lipid species in plasma, kidney, retina and nerve in the BKS $d b / d b$ mouse [30]. In doing so, we uncovered tissue-specific alterations in several lipid classes, both in their level and directionality, which were defined by class, carbon side-chain length and saturation levels (Fig. 2). While alterations in individual lipids were mostly tissue-dependent, the kidneys and nerves from 


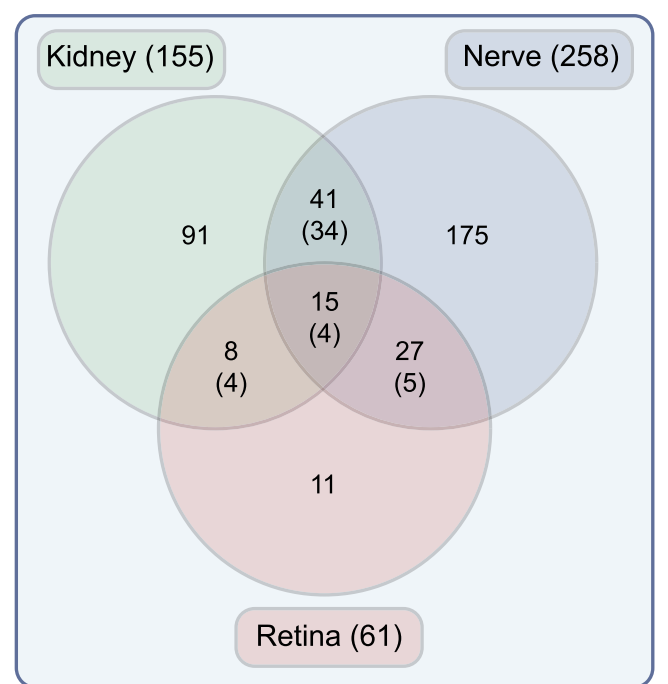

Fig. 2 Diabetes is associated with tissue-specific lipid alterations. The Venn diagram represents significantly altered lipid features observed in the BKS $d b / d b$ mouse model of diabetes compared with control. Only 15 lipid features were significantly altered across complication-prone tissues, including diacylglycerol, phospholipids and cardiolipins. The direction of change in these shared features varied across different tissues with only four of the 15 common to all three tissues changing in the same direction (number of features with common direction is shown in parentheses), further demonstrating the tissue-specific nature of the lipid alterations associated with diabetes. Data derived from [30]. This figure is available as part of a downloadable slideset

diabetic mouse models exhibited an overall trend towards increased levels of numerous lipid species. In contrast, overall lipid content in the retinas of diabetic mice was generally decreased. In line with these findings, extensive lipid accumulation has been reported within kidney glomeruli and tubulointerstitium in individuals with type 2 diabetes who had diabetic kidney disease [63]. Moreover, using postmortem human retina samples, we recently obtained data suggesting alterations in lipid metabolism in retinas from donors with diabetic retinopathy (P. E. Fort, unpublished data), consistent with the reduction observed in BKS $d b / d b$ mice [30]. The importance of these findings is highlighted by the observation that genetic deletion of the fatty acid synthase gene in the neuroretina leads to progressive neurodegeneration, with synaptic dysfunction, neuronal cell apoptosis and visual impairment [64]. As these pathological changes are also observed in diabetic retinopathy, the possibility arises that impaired lipid synthesis may contribute to the neurodegenerative effects of diabetes on the retina. Similarly, transcriptomic analysis showed altered lipid metabolism in human sural nerve biopsies obtained from diabetic individuals with progressive vs stable diabetic neuropathy $[34,65]$. Taken together, these findings suggest a complicated balance and interaction between systemic and tissue-specific lipid levels and warrant further analysis of the relative importance of dyslipidaemia and tissue-specific lipid metabolism in the pathogenesis of diabetic complications.
Fatty acid regulation Insulin directly regulates circulating fatty acids by controlling the conversion of non-esterified fatty acids to triacylglycerol and its subsequent secretion from the liver. As such, diabetes, low insulin levels and insulin resistance disrupt plasma fatty acid and complex lipid composition [66]. Insulin resistance is linked to changes in levels of several lipid classes, including triacylglycerols, while diabetes risk correlates with lower fatty acid carbon number and lower degree of fatty acid saturation [67]. Recently, lipidomic analysis was used to associate plasma fatty acid and triacylglycerol composition with progression of kidney disease in diabetes $[27,29]$. Of note, as diabetic kidney disease progresses and the glomerular filtration barrier deteriorates, the kidney is likely to encounter higher levels of albumin-bound fatty acids in the filtrate, potentially increasing the impact of dyslipidaemia on tubular cells.

In the retina, highly enriched polyunsaturated fatty acids (PUFAs) have been intensely studied in the context of their role in regulating vascular function and angiogenesis [68]. In models associated with late stages of diabetic retinopathy, lipidomic analyses identified a reduction in some of the largest n-3 PUFAs [68], while transcriptomics revealed reduced expression of the enzymes involved in PUFA synthesis [69]. Interestingly, dietary manipulations focusing on PUFAs caused a positive impact on pathological retinal neovascularisation in an animal model of oxygen-induced retinopathy. This effect may be related to the role of PUFAs in inflammation [70] or in the synthesis of very-long-chain ceramides, which seem to maintain vascular integrity in experimental diabetes [71].

Diabetic neuropathy, of the three complications, most closely associates with dyslipidaemia [16]. Obesity induced by a diet rich in saturated fatty acids is associated with the development of diabetic neuropathy in individuals with type 2 diabetes [72] and, as with diabetic retinopathy, PUFA supplementation ameliorates symptoms of diabetic neuropathy [73]. This clinical finding is strongly supported by the preclinical work of Yorek and colleagues (reviewed in [74]), that shows clinical benefit of PUFA treatment in multiple rodent models of diabetic neuropathy. Moreover, transcriptomic analysis reveals an upregulation in fatty acid metabolism in the nerves of BKS $d b / d b$ mice with diabetic neuropathy [75]. In cellular studies, high concentrations of the saturated fatty acid palmitate impair sensory neuron mitochondrial trafficking and alter mitochondrial energy production [76]. However, supplementation of cultured neurons with monounsaturated fatty acids such as oleate prevents palmitateinduced mitochondrial abnormalities through lipid droplet formation [77]. We also found that cultured Schwann cells increase the expression of medium-to-long-chain acylcarnitines in response to long-chain fatty acid treatment. Accumulation of these acylcarnitines has been associated with neurotoxicity and insulin resistance $[78,79]$. 
Collectively, these preclinical and clinical studies suggest an important role for fatty acid dysregulation in all three diabetic complications. Overall, saturated fatty acids are damaging to complication-prone tissues, while supplementation with PUFAs may restore normal function and provide a treatment modality. While more research is needed, the concept of 'bad' (saturated) and 'good' (unsaturated) fatty acids holds true at the present time.

Lipid $\beta$-oxidation Peroxisome proliferator-activated receptors (PPARs) and PPAR, gamma, coactivator 1, alpha (PGC1- $\alpha$ ) play a key role in the regulation of fatty acid oxidation as they regulate the expression of proteins responsible for fatty acid uptake, such as the multifunctional fatty acid translocase CD36. In animal models and humans, PPAR signalling is highly dysregulated in diabetes $[34,75,80,81]$. CD36 is upregulated in kidneys of diabetic animals and individuals with diabetes $[63,82]$, and is involved in mediating the apoptotic and oxidative effects of oxidised LDL immune complexes in metabolically challenged human retinal pericytes [83]. Similarly, CD36 expression is increased in peripheral nerves of BKS $d b / d b$ mice and individuals with progressive diabetic neuropathy $[34,75]$.

Fatty acid profiles in tissues not only depend on plasma levels and uptake capacity, as discussed above, but also on the local lipid metabolism in these tissues. We hypothesised that, like glucose, there would be tissue-specific alterations in lipid $\beta$-oxidation with diabetes. Indeed, using a combination of transcriptomic and metabolomic approaches to assess the impact of type 2 diabetes on $\beta$-oxidation, we demonstrated a tissue-specific response of this pathway in BKS $d b / d b$ mice [26]. Whereas transcriptomic analyses revealed increased expression of genes for fatty acid oxidation enzymes in the diabetic kidney and nerve, diabetes increased levels of the metabolites associated with fatty acid metabolism and $\beta$ oxidation in the kidney and retina and decreased these metabolites in peripheral nerves. The use of palmitic acid labelled with the stable isotope ${ }^{13} \mathrm{C}$ to analyse the effects of type 2 diabetes on the metabolic flux through fatty acid catabolism showed increased palmitate uptake in all three tissues. Moreover, the analysis confirmed a progressive increase in $\beta$-oxidation in the kidney only, while suggesting incomplete fatty acid catabolism in retina and peripheral nerves. Consistent with these findings, Schwann cells exposed to high levels of long-chain fatty acids undergo incomplete fatty acid catabolism. This leads to altered acylcarnitine accumulation, which is known to have neurotoxic properties [84], as discussed earlier in this review.

We observed increased kidney $\beta$-oxidation in a preclinical murine model of diabetic kidney disease, while transcriptomic analysis using kidney tissue from individuals with chronic kidney disease revealed decreased expression of fatty acid oxidation enzymes in the presence of fibrosis [85]. This dichotomy suggests that lipid metabolism may be differentially regulated throughout disease progression, something that has not been thoroughly examined in each of the complication-prone tissues. On a tissue-specific level, lipid $\beta$-oxidation may regulate tissue function by inducing epigenetic changes [86] and metabolic intermediates of lipid $\beta$ oxidation, such as succinate and fumarate, have been shown to stimulate pathogenic pathways $[87,88]$. Additionally, lipid $\beta$-oxidation (especially of very-long-chain fatty acids) may mediate diabetes-induced increases in oxidative stress, potentially to a greater degree than increased glucose catabolism, thus driving diabetic complications $[89,90]$.

Lipids and mitochondrial dysfunction Given their role in energy metabolism, mitochondria are presumably a key interaction point between glucose metabolism, lipid metabolism and diabetic complications. In addition to undergoing $\beta$-oxidation in mitochondria (as discussed above), lipids also directly affect mitochondrial function (reviewed in [91]). Cardiolipins (inner mitochondrial membrane lipids) are important for mitochondrial structure and mitochondrial metabolism. We recently identified an increase in immature cardiolipins in the diabetic kidney [30], which is associated with reduced bioenergetics [92], a hallmark of diabetic kidney disease [93]. Interestingly, preserving mitochondrial structure with a cardiolipin-binding peptide maintains mitochondrial function in the kidney $[94,95]$, reverses visual decline in diabetic mice [96] and improves bioenergetics and mitochondrial trafficking in neurons [97]. Additionally, exposure of Schwann cells to a mixture of long-chain fatty acids similar to that observed under diabetic conditions impairs mitochondrial function and induces oxidative stress [84]. As discussed above, saturated fatty acids damage sensory axons by depolarising neuronal mitochondria and impairing mitochondrial trafficking [76]. Supplementation with monounsaturated fatty acids prevents mitochondrial dysfunction through lipid droplet formation, highlighting again a complex role for lipid products and metabolism in diabetes complications [77].

Lipids in inflammation Integration of omics studies have revealed correlations, if not causal relationships, between altered lipid metabolism and inflammation. PUFAs, especially $n-3$ PUFAs, can regulate inflammation by inhibition of NF-KB activation and nuclear translocation [98, 99]. In contrast, $n-6$ PUFAs are precursors of bioactive lipids such as arachidonic acid, which would itself promote a proinflammatory environment and development of diabetic complications. Elevated arachidonic acid-derived eicosanoids in serum independently predict diabetic kidney disease progression [29], while altered plasma fatty acid composition is associated with retinal and systemic inflammation [69]. Multiple studies suggest that dietary manipulations that increase the ratio of $n-3$ to $n-6$ PUFAs, decrease the risk of diabetic complications in adult 
participants with type 2 diabetes [100-102]. Lipid levels are dependent on multiple mechanisms of regulation including dietary intake and de-novo synthesis, and also on the exchange and recycling systems. Among those regulatory mechanisms, reverse cholesterol transport and its regulation by specific receptors, namely the farnesoid X receptor (FXR) and the liver X receptor (LXR), has been receiving increasing attention in relation to inflammation, especially in the pathogenesis of diabetic kidney disease $[103,104]$ and diabetic retinopathy [105]. LXR activation was also shown to improve peripheral nerve function in rodents with obesity and diabetes-induced neuropathy, specifically by reducing endoplasmic reticulum stress or restoring myelin lipid composition; however, little is known about the anti-inflammatory effects of LXR in the setting of neuropathy $[106,107]$. These studies are intriguing and certainly warrant further exploration of the implication of these mechanisms in the onset and progression of diabetic complications.

Other aspects of lipid metabolism have been suggested to impact systemic as well as local inflammation. Multiple studies using unbiased approaches analysing the transcriptome, proteome or metabolome of various tissues affected by diabetes have reported lipid metabolism and inflammation as being among the top pathways affected $[26,85,108-111]$. These studies in animal models of diabetes are now complemented and supported by studies in individuals with diabetes. For example, we recently used transcriptomic analysis to demonstrate that abnormal lipid metabolism and inflammation in peripheral nerves are conserved across multiple mouse models of type 2 diabetes and in individuals with progressive diabetic neuropathy [65]. Moreover, several studies found positive associations between serum markers of inflammation, lipid metabolism and onset and progression of complications in individuals with type 1 and type 2 diabetes $[29,112,113]$.

\section{Treatment of diabetic complications: one size does not fit all}

Tissues with distinct structures and functions are affected by diabetes, suggesting the involvement of tissue-specific mechanisms in the pathogenesis of the disease. In the kidney, metabolism is associated with haemodynamic factors and diminishing filtration, which allow waste products to accumulate in blood [114]. In the retina, metabolic alterations have been proposed to be responsible for dysregulation of the glutamine-glutamate cycle in retinal Müller glial cells, an effect believed to be associated with glutamate excitotoxicity and cell death [115]. In the peripheral nerves, metabolic irregularities in Schwann cells are thought to induce mitochondrial deficits, accumulation of neurotoxic species and transfer of lipotoxic species to axons, a series of injurious events that may lead to axonal degeneration $[24,79]$. Altogether, these studies, while not being exhaustive, highlight once more some of the 'tissue/cell' specificity of the impact of diabetes and emphasise the need for global analysis to assess the tissuespecific mechanisms at play in order to develop targeted interventions.

\section{Conclusions}

While it is tempting to combine 'microvascular' complications under one mechanism, it is clear that diabetes dysregulates metabolism in a tissue-specific manner. A role for generalised mechanisms cannot be fully excluded but the current body of knowledge strongly suggests that tissuespecific metabolic alterations, rather than a single unifying metabolic mechanism, are driving the onset and progression of diabetic complications. Metabolic dysregulation includes not only hyperglycaemia but also alterations in systemic and local lipid metabolism. Therapeutic development for diabetic complications will require a better understanding of the crosstalk between glucose and lipid metabolism in individual tissues, if not individual cell types, which most likely differs in each tissue despite the common underlying condition. Additionally, while the large amount of information gathered from numerous animal models of diabetes relative to the pathophysiology of these complications has been critical, difficulties associated with translation still highlight the need for human-based studies. Discovery approaches, especially those performed in human tissues across the gene-transcript-protein-metabolite continuum, have already demonstrated a potential for identifying tissue-specific adaptations and will continue to be the starting point in unveiling the mechanisms of diabetic complications and identifying novel therapeutic targets. Ultimately, a better integration and communication between researchers working on the respective complications, combined with a broader use of human samples and clinical data, is necessary to perform integrated analysis of complications pathophysiology, characterise their respective mechanisms and define new treatment options.

Funding Work in the authors laboratories is supported by the National Institutes of Health (1R24082841 to SFA, ELF, TWG, SP and PEF; P30DK081943 to SP; R01EY020582 to SFA, TWG and PEF; R01EY029349 and R01EY020823 to SFA; R01EY020895 to PEF; P30EY007003 Kellogg Eye Center Core Center for Vision Research to SFA, TWG and PEF; R24DK08284, P30DK081943 and P30DK89503 to KMS and SP), the Novo Nordisk Foundation (NNF14OC0011633 to ELF and SE), Milstein, Nathan and Rose Research Fund (to SE) and the Program for Neurology Research \& Discovery (to ELF and SE).

Duality of interest The authors declare that there is no duality of interest associated with this manuscript.

Contribution statement All authors were responsible for drafting the article and revising it critically for important intellectual content. All authors approved the version to be published. 


\section{References}

1. International Diabetes Federation (2017) Diabetes facts and figures. Available from https://idf.org/aboutdiabetes/what-isdiabetes/facts-figures.html. Accessed 11 July 2019

2. Imperatore G, Boyle JP, Thompson TJ et al (2012) Projections of type 1 and type 2 diabetes burden in the U.S. population aged $<20$ years through 2050: dynamic modeling of incidence, mortality, and population growth. Diabetes Care 35(12):2515-2520. https://doi.org/10.2337/dc12-0669

3. Mayer-Davis EJ, Lawrence JM, Dabelea D et al (2017) Incidence Trends of Type 1 and Type 2 Diabetes among Youths, 2002-2012. N Engl J Med 376(15):1419-1429. https://doi.org/10.1056/ NEJMoa1610187

4. Forbes JM, Cooper ME (2013) Mechanisms of diabetic complications. Physiol Rev 93(1):137-188. https://doi.org/10.1152/ physrev.00045.2011

5. United States Renal Data System (2018) 2018 annual data report. Available from www.usrds.org/adrhighlights.aspx. Accessed 11 July 2019

6. Persson F, Rossing P (2018) Diagnosis of diabetic kidney disease: state of the art and future perspective. Kidney Int Suppl 8(1):2-7. https://doi.org/10.1016/j.kisu.2017.10.003

7. Choudhury D, Tuncel M, Levi M (2010) Diabetic nephropathy a multifaceted target of new therapies. Discov Med 10(54):406415

8. Yau JW, Rogers SL, Kawasaki R et al (2012) Global prevalence and major risk factors of diabetic retinopathy. Diabetes Care 35(3): 556-564. https://doi.org/10.2337/dc11-1909

9. Abcouwer SF, Gardner TW (2014) Diabetic retinopathy: loss of neuroretinal adaptation to the diabetic metabolic environment. Ann N Y Acad Sci 1311(1):174-190. https://doi.org/10.1111/ nyas. 12412

10. Duh EJ, Sun JK, Stitt AW (2017) Diabetic retinopathy: current understanding, mechanisms, and treatment strategies. JCI Insight 2(14):e93751. https://doi.org/10.1172/jci.insight.93751

11. Rubsam A, Parikh S, Fort PE (2018) Role of Inflammation in Diabetic Retinopathy. Int J Mol Sci 19(4):942. https://doi.org/10. 3390/ijms19040942

12. Callaghan BC, Price RS, Feldman EL (2015) Distal Symmetric Polyneuropathy: A Review. JAMA 314(20):2172-2181. https:// doi.org/10.1001/jama.2015.13611

13. Feldman EL, Nave KA, Jensen TS, Bennett DLH (2017) New Horizons in Diabetic Neuropathy: Mechanisms, Bioenergetics, and Pain. Neuron 93(6):1296-1313. https://doi.org/10.1016/j. neuron.2017.02.005

14. Dyck PJ, Giannini C (1996) Pathologic alterations in the diabetic neuropathies of humans: a review. J Neuropathol Exp Neurol 55(12):1181-1193. https://doi.org/10.1097/00005072199612000-00001

15. Shamoon H, Duffy H, Fleischer N et al (1993) The Effect of Intensive Treatment of Diabetes on the Development and Progression of Long-Term Complications in Insulin-Dependent Diabetes-Mellitus. N Engl J Med 329:977-986

16. Callaghan BC, Little AA, Feldman EL, Hughes RA (2012) Enhanced glucose control for preventing and treating diabetic neuropathy. Cochrane Database Syst Rev 6:CD007543. https:// doi.org/10.1002/14651858.CD007543.pub2

17. King P, Peacock I, Donnelly R (1999) The UK Prospective Diabetes Study (UKPDS): clinical and therapeutic implications for type 2 diabetes. Br J Clin Pharmacol 48:643-648

18. Gaede PH, Jepsen PV, Larsen JN, Jensen GV, Parving HH, Pedersen OB (2003) The Steno-2 study. Intensive multifactorial intervention reduces the occurrence of cardiovascular disease in patients with type 2 diabetes [article in Danish]. Ugeskr Laeger 165(26):2658-2661
19. Duckworth W, Abraira C, Moritz T et al (2009) Glucose control and vascular complications in veterans with type 2 diabetes. $\mathrm{N}$ Eng1 J Med 360(2):129-139. https://doi.org/10.1056/ NEJMoa0808431

20. Terry T, Raravikar K, Chokrungvaranon N, Reaven PD (2012) Does aggressive glycemic control benefit macrovascular and microvascular disease in type 2 diabetes? Insights from ACCORD, ADVANCE, and VADT. Curr Cardiol Rep 14(1):79-88. https:// doi.org/10.1007/s11886-011-0238-6

21. Zoungas S, Arima H, Gerstein HC et al (2017) Effects of intensive glucose control on microvascular outcomes in patients with type 2 diabetes: a meta-analysis of individual participant data from randomised controlled trials. Lancet Diabetes Endocrinol 5(6): 431-437. https://doi.org/10.1016/S2213-8587(17)30104-3

22. Callaghan BC, Xia R, Banerjee M et al (2016) Metabolic Syndrome Components Are Associated With Symptomatic Polyneuropathy Independent of Glycemic Status. Diabetes Care 39(5):801-807. https://doi.org/10.2337/dc16-0081

23. Giacco F, Brownlee M (2010) Oxidative stress and diabetic complications. Circ Res 107(9):1058-1070. https://doi.org/10.1161/ CIRCRESAHA.110.223545

24. Feldman AL, Griffin SJ, Fharm E et al (2017) Screening for type 2 diabetes: do screen-detected cases fare better? Diabetologia 60(11):2200-2209. https://doi.org/10.1007/s00125-017-4402-4

25. Tang WH, Martin KA, Hwa J (2012) Aldose reductase, oxidative stress, and diabetic mellitus. Front Pharmacol 3:87

26. Sas KM, Kayampilly P, Byun J et al (2016) Tissue-specific metabolic reprogramming drives nutrient flux in diabetic complications. JCI Insight 1:e86976

27. Afshinnia F, Rajendiran TM, Karnovsky A et al (2016) Lipidomic Signature of Progression of Chronic Kidney Disease in the Chronic Renal Insufficiency Cohort. Kidney Int Rep 1(4):256 268. https://doi.org/10.1016/j.ekir.2016.08.007

28. Afshinnia F, Rajendiran TM, Soni T et al (2018) Impaired betaOxidation and Altered Complex Lipid Fatty Acid Partitioning with Advancing CKD. J Am Soc Nephrol 29(1):295-306. https://doi.org/10.1681/ASN.2017030350

29. Afshinnia F, Rajendiran TM, Wernisch S et al (2018) Lipidomics and Biomarker Discovery in Kidney Disease. Semin Nephrol 38(2):127-141. https://doi.org/10.1016/j.semnephrol.2018.01. 004

30. Sas KM, Lin J, Rajendiran TM et al (2018) Shared and distinct lipid-lipid interactions in plasma and affected tissues in a diabetic mouse model. J Lipid Res 59(2):173-183. https://doi.org/10.1194/ jlr.M077222

31. Sas KM, Nair V, Byun J et al (2015) Targeted Lipidomic and Transcriptomic Analysis Identifies Dysregulated Renal Ceramide Metabolism in a Mouse Model of Diabetic Kidney Disease. J Proteomics Bioinform s14(Suppl 14). https://doi.org/10.4172/ jpb.S14-002

32. Freeman OJ, Unwin RD, Dowsey AW et al (2016) Metabolic Dysfunction Is Restricted to the Sciatic Nerve in Experimental Diabetic Neuropathy. Diabetes 65(1):228-238. https://doi.org/10. 2337/db15-0835

33. Priyadarsini S, McKay TB, Sarker-Nag A et al (2016) Complete metabolome and lipidome analysis reveals novel biomarkers in the human diabetic corneal stroma. Exp Eye Res 153:90-100. https:// doi.org/10.1016/j.exer.2016.10.010

34. Hur J, Sullivan KA, Pande M et al (2011) The identification of gene expression profiles associated with progression of human diabetic neuropathy. Brain 134(11):3222-3235. https://doi.org/ 10.1093/brain/awr228

35. Chen SC, Tseng CH (2013) Dyslipidemia, kidney disease, and cardiovascular disease in diabetic patients. Rev Diabet Stud 10(2-3):88-100. https://doi.org/10.1900/RDS.2013.10.88 
36. Dronavalli S, Duka I, Bakris GL (2008) The pathogenesis of diabetic nephropathy. Nat Clin Pract Endocrinol Metab 4(8):444 452. https://doi.org/10.1038/ncpendmet0894

37. Maqbool M, Cooper ME, Jandeleit-Dahm KAM (2018) Cardiovascular Disease and Diabetic Kidney Disease. Semin Nephrol 38(3):217-232. https://doi.org/10.1016/j.semnephrol. 2018.02.003

38. Davis TM, Ting R, Best JD et al (2011) Effects of fenofibrate on renal function in patients with type 2 diabetes mellitus: the Fenofibrate Intervention and Event Lowering in Diabetes (FIELD) Study. Diabetologia 54(2):280-290. https://doi.org/10. 1007/s00125-010-1951-1

39. Peng H, Luo P, Li Y et al (2013) Simvastatin alleviates hyperpermeability of glomerular endothelial cells in early-stage diabetic nephropathy by inhibition of RhoA/ROCK1. PLoS One 8(11):e80009. https://doi.org/10.1371/journal.pone.0080009

40. Sandhu S, Wiebe N, Fried LF, Tonelli M (2006) Statins for improving renal outcomes: a meta-analysis. J Am Soc Nephrol 17(7): 2006-2016. https://doi.org/10.1681/ASN.2006010012

41. Colhoun HM, Betteridge DJ, Durrington PN et al (2009) Effects of atorvastatin on kidney outcomes and cardiovascular disease in patients with diabetes: an analysis from the Collaborative Atorvastatin Diabetes Study (CARDS). Am J Kidney Dis 54(5): 810-819. https://doi.org/10.1053/j.ajkd.2009.03.022

42. Rahman M, Baimbridge C, Davis BR et al (2008) Progression of kidney disease in moderately hypercholesterolemic, hypertensive patients randomized to pravastatin versus usual care: a report from the Antihypertensive and Lipid-Lowering Treatment to Prevent Heart Attack Trial (ALLHAT). Am J Kidney Dis 52(3):412424. https://doi.org/10.1053/j.ajkd.2008.05.027

43. Giunti S, Calkin AC, Forbes JM et al (2010) The pleiotropic actions of rosuvastatin confer renal benefits in the diabetic ApoE knockout mouse. Am J Physiol Renal Physiol 299(3):F528F535. https://doi.org/10.1152/ajprenal.00127.2010

44. Chew EY, Klein ML, Ferris FL 3rd et al (1996) Association of elevated serum lipid levels with retinal hard exudate in diabetic retinopathy. Early Treatment Diabetic Retinopathy Study (ETDRS) Report 22. Arch Ophthalmol 114(9):1079-1084. https://doi.org/10.1001/archopht.1996.01100140281004

45. Klein BE, Myers CE, Howard KP, Klein R (2015) Serum Lipids and Proliferative Diabetic Retinopathy and Macular Edema in Persons With Long-term Type 1 Diabetes Mellitus: The Wisconsin Epidemiologic Study of Diabetic Retinopathy. JAMA Ophthalmol 133(5):503-510. https://doi.org/10.1001/ jamaophthalmol.2014.5108

46. Romero P, Salvat M, Fernandez J, Baget M, Martinez I (2007) Renal and retinal microangiopathy after 15 years of follow-up study in a sample of Type 1 diabetes mellitus patients. J Diabetes Complicat 21(2):93-100. https://doi.org/10.1016/j. jdiacomp.2006.04.001

47. Gupta A, Gupta V, Thapar S, Bhansali A (2004) Lipid-lowering drug atorvastatin as an adjunct in the management of diabetic macular edema. Am J Ophthalmol 137(4):675-682. https://doi. org/10.1016/j.ajo.2003.11.017

48. Sen K, Misra A, Kumar A, Pandey RM (2002) Simvastatin retards progression of retinopathy in diabetic patients with hypercholesterolemia. Diabetes Res Clin Pract 56(1):1-11. https://doi.org/10. 1016/S0168-8227(01)00341-2

49. Colhoun HM, Betteridge DJ, Durrington PN et al (2004) Primary prevention of cardiovascular disease with atorvastatin in type 2 diabetes in the Collaborative Atorvastatin Diabetes Study (CARDS): multicentre randomised placebo-controlled trial. Lancet 364(9435):685-696. https://doi.org/10.1016/S01406736(04)16895-5

50. Heart Protection Study Collaborative Group (2003) MRC/BHF Heart Protection Study of cholesterol-lowering with simvastatin in 5963 people with diabetes: a randomised placebo-controlled trial. Lancet 361:2005-2016

51. Kang EY, Chen TH, Garg SJ et al (2019) Association of Statin Therapy With Prevention of Vision-Threatening Diabetic Retinopathy. JAMA Ophthalmol 137(4):363-371. https://doi. org/10.1001/jamaophthalmol.2018.6399

52. ACCORD Study Group, ACCORD Eye Study Group (2010) Effects of medical therapies on retinopathy progression in type 2 diabetes. N Engl J Med 363(3):233-244. https://doi.org/10.1056/ NEJMoa1001288

53. Keech AC, Mitchell P, Summanen PA et al (2007) Effect of fenofibrate on the need for laser treatment for diabetic retinopathy (FIELD study): a randomised controlled trial. Lancet 370(9600): 1687-1697. https://doi.org/10.1016/S0140-6736(07)61607-9

54. Zhou Y, Wang C, Shi K, Yin X (2018) Relationship between dyslipidemia and diabetic retinopathy: a systematic review and meta-analysis. Medicine 97(36):e12283. https://doi.org/10.1097/ MD.0000000000012283

55. Liu Q, Zhang X, Cheng R, Ma JX, Yi J, Li J (2019) Salutary effect of fenofibrate on type 1 diabetic retinopathy via inhibiting oxidative stress-mediated $\mathrm{Wnt} / \beta$-catenin pathway activation. Cell Tissue Res 376(2):165-177. https://doi.org/10.1007/s00441-0182974-z

56. Pearsall EA, Cheng R, Matsuzaki S et al (2019) Neuroprotective effects of PPARalpha in retinopathy of type 1 diabetes. PLoS One 14(2):e0208399. https://doi.org/10.1371/journal.pone.0208399

57. Wang N, Zou C, Zhao S, Wang Y, Han C, Zheng Z (2018) Fenofibrate Exerts Protective Effects in Diabetic Retinopathy via Inhibition of the ANGPTL3 Pathway. Invest Ophthalmol Vis Sci 59(10):4210-4217. https://doi.org/10.1167/iovs. 18-24155

58. Liu Q, Zhang F, Zhang X et al (2018) Fenofibrate ameliorates diabetic retinopathy by modulating Nrf2 signaling and NLRP3 inflammasome activation. Mol Cell Biochem 445(1-2):105-115. https://doi.org/10.1007/s11010-017-3256-x

59. Smith AG, Singleton JR (2013) Obesity and hyperlipidemia are risk factors for early diabetic neuropathy. J Diabetes Complicat 27(5):436-442. https://doi.org/10.1016/j.jdiacomp.2013.04.003

60. Wiggin TD, Sullivan KA, Pop-Busui R, Amato A, Sima AA, Feldman EL (2009) Elevated triglycerides correlate with progression of diabetic neuropathy. Diabetes 58(7):1634-1640. https:// doi.org/10.2337/db08-1771

61. Rajamani K, Colman PG, Li LP et al (2009) Effect of fenofibrate on amputation events in people with type 2 diabetes mellitus (FIELD study): a prespecified analysis of a randomised controlled trial. Lancet 373(9677):1780-1788. https://doi.org/10.1016/ S0140-6736(09)60698-X

62. Vincent AM, Hayes JM, McLean LL, Vivekanandan-Giri A, Pennathur S, Feldman EL (2009) Dyslipidemia-induced neuropathy in mice: the role of oxLDL/LOX-1. Diabetes 58(10):23762385. https://doi.org/10.2337/db09-0047

63. Herman-Edelstein M, Scherzer P, Tobar A, Levi M, Gafter U (2014) Altered renal lipid metabolism and renal lipid accumulation in human diabetic nephropathy. J Lipid Res 55(3):561-572. https://doi.org/10.1194/jlr.P040501

64. Rajagopal R, Zhang S, Wei X et al (2018) Retinal de novo lipogenesis coordinates neurotrophic signaling to maintain vision. JCI Insight 3(1):e97076. https://doi.org/10.1172/jci.insight.97076

65. McGregor BA, Eid S, Rumora AE et al (2018) Conserved Transcriptional Signatures in Human and Murine Diabetic Peripheral Neuropathy. Sci Rep 8(1):17678. https://doi.org/10. 1038/s41598-018-36098-5

66. Goldberg IJ (2001) Diabetic dyslipidemia: causes and consequences. J Clin Endocrinol Metab 86(3):965-971. https://doi. org/10.1210/jcem.86.3.7304

67. Rhee EP, Cheng S, Larson MG et al (2011) Lipid profiling identifies a triacylglycerol signature of insulin resistance and improves 
diabetes prediction in humans. J Clin Invest 121(4):1402-1411. https://doi.org/10.1172/JCI44442

68. Gong Y, Fu Z, Liegl R, Chen J, Hellstrom A, Smith LE (2017) $\omega$ 3 and $\omega-6$ long-chain PUFAs and their enzymatic metabolites in neovascular eye diseases. Am J Clin Nutr 106(1):16-26. https:// doi.org/10.3945/ajen.117.153825

69. Tikhonenko M, Lydic TA, Wang Y et al (2010) Remodeling of retinal Fatty acids in an animal model of diabetes: a decrease in long-chain polyunsaturated fatty acids is associated with a decrease in fatty acid elongases Elov12 and Elov14. Diabetes 59(1): 219-227. https://doi.org/10.2337/db09-0728

70. Connor KM, SanGiovanni JP, Lofqvist C et al (2007) Increased dietary intake of omega-3-polyunsaturated fatty acids reduces pathological retinal angiogenesis. Nat Med 13(7):868-873. https://doi.org/10.1038/nm1591

71. Kady NM, Liu X, Lydic TA et al (2018) ELOVL4-Mediated Production of Very Long-Chain Ceramides Stabilizes Tight Junctions and Prevents Diabetes-Induced Retinal Vascular Permeability. Diabetes 67(4):769-781. https://doi.org/10.2337/ db17-1034

72. Callaghan BC, Gao LL, Li YF et al (2018) Diabetes and obesity are the main metabolic drivers of peripheral neuropathy. Ann Clin Transl Neur 5(4):397-405. https://doi.org/10.1002/acn3.531

73. Duran AM, Salto LM, Camara J et al (2019) Effects of omega-3 polyunsaturated fatty-acid supplementation on neuropathic pain symptoms and sphingosine levels in Mexican-Americans with type 2 diabetes. Diabetes Metab Syndr Obes 12:109-120. https://doi.org/10.2147/DMSO.S187268

74. Yorek MA (2018) The Potential Role of Fatty Acids in Treating Diabetic Neuropathy. Curr Diab Rep 18(10):86. https://doi.org/10. 1007/s11892-018-1046-9

75. Pande M, Hur J, Hong Y et al (2011) Transcriptional profiling of diabetic neuropathy in the BKS db/db mouse: a model of type 2 diabetes. Diabetes 60(7):1981-1989. https://doi.org/10.2337/ $\mathrm{db} 10-1541$

76. Rumora AE, Lentz SI, Hinder LM et al (2018) Dyslipidemia impairs mitochondrial trafficking and function in sensory neurons. FASEB J 32(1):195-207. https://doi.org/10.1096/fj.201700206R

77. Kwon B, Lee HK, Querfurth HW (2014) Oleate prevents palmitate-induced mitochondrial dysfunction, insulin resistance and inflammatory signaling in neuronal cells. Biochim Biophys Acta 1843(7):1402-1413. https://doi.org/10.1016/j.bbamcr.2014. 04.004

78. Schooneman MG, Vaz FM, Houten SM, Soeters MR (2013) Acylcarnitines: reflecting or inflicting insulin resistance? Diabetes 62(1):1-8. https://doi.org/10.2337/db12-0466

79. Viader A, Sasaki Y, Kim S et al (2013) Aberrant Schwann cell lipid metabolism linked to mitochondrial deficits leads to axon degeneration and neuropathy. Neuron 77(5):886-898. https://doi. org/10.1016/j.neuron.2013.01.012

80. Ding L, Cheng R, Hu Y et al (2014) Peroxisome proliferatoractivated receptor alpha protects capillary pericytes in the retina. Am J Pathol 184(10):2709-2720. https://doi.org/10.1016/j.ajpath. 2014.06.021

81. Guan Y (2004) Peroxisome proliferator-activated receptor family and its relationship to renal complications of the metabolic syndrome. J Am Soc Nephrol 15(11):2801-2815. https://doi.org/10. 1097/01.ASN.0000139067.83419.46

82. Ansquer JC, Foucher C, Rattier S, Taskinen MR, Steiner G (2005) Fenofibrate reduces progression to microalbuminuria over 3 years in a placebo-controlled study in type 2 diabetes: results from the Diabetes Atherosclerosis Intervention Study (DAIS). Am J Kidney Dis 45(3):485-493. https://doi.org/10.1053/j.ajkd.2004. 11.004

83. Fu D, Yu JY, Wu M et al (2014) Immune complex formation in human diabetic retina enhances toxicity of oxidized LDL towards retinal capillary pericytes. J Lipid Res 55(5):860-869. https://doi. org/10.1194/jlr.M045401

84. Hinder LM, Figueroa-Romero C, Pacut C et al (2014) Long-chain acyl coenzyme A synthetase 1 overexpression in primary cultured Schwann cells prevents long chain fatty acid-induced oxidative stress and mitochondrial dysfunction. Antioxid Redox Signal 21(4):588-600. https://doi.org/10.1089/ars.2013.5248

85. Kang HM, Ahn SH, Choi P et al (2015) Defective fatty acid oxidation in renal tubular epithelial cells has a key role in kidney fibrosis development. Nat Med 21(1):37-46. https://doi.org/10. 1038/nm.3762

86. Yuan H, Reddy MA, Deshpande S et al (2016) Epigenetic Histone Modifications Involved in Profibrotic Gene Regulation by 12/15Lipoxygenase and Its Oxidized Lipid Products in Diabetic Nephropathy. Antioxid Redox Signal 24(7):361-375. https://doi. org/10.1089/ars.2015.6372

87. He W, Miao FJ, Lin DC et al (2004) Citric acid cycle intermediates as ligands for orphan G-protein-coupled receptors. Nature 429(6988):188-193. https://doi.org/10.1038/nature02488

88. You YH, Quach T, Saito R, Pham J, Sharma K (2016) Metabolomics Reveals a Key Role for Fumarate in Mediating the Effects of NADPH Oxidase 4 in Diabetic Kidney Disease. J Am Soc Nephrol 27(2):466-481. https://doi.org/10.1681/ASN. 2015030302

89. Joyal JS, Sun Y, Gantner ML et al (2016) Retinal lipid and glucose metabolism dictates angiogenesis through the lipid sensor Ffar1. Nat Med 22(4):439-445. https://doi.org/10.1038/nm.4059

90. Willems D, Dorchy H, Dufrasne D (1998) Serum antioxidant status and oxidized LDL in well-controlled young type 1 diabetic patients with and without subclinical complications. Atherosclerosis 137(Suppl 1):S61-S64. https://doi.org/10.1016/ S0021-9150(97)00320-1

91. Martensson CU, Doan KN, Becker T (2017) Effects of lipids on mitochondrial functions. Biochim Biophys Acta Mol Cell Biol Lipids 1862(1):102-113. https://doi.org/10.1016/j.bbalip.2016. 06.015

92. Chicco AJ, Sparagna GC (2007) Role of cardiolipin alterations in mitochondrial dysfunction and disease. Am J Physiol Cell Physiol 292(1):C33-C44. https://doi.org/10.1152/ajpcell.00243.2006

93. Darshi M, Van Espen B, Sharma K (2016) Metabolomics in Diabetic Kidney Disease: Unraveling the Biochemistry of a Silent Killer. Am J Nephrol 44(2):92-103. https://doi.org/10. 1159/000447954

94. Szeto HH, Liu S, Soong Y et al (2017) Mitochondria Protection after Acute Ischemia Prevents Prolonged Upregulation of IL- $1 \beta$ and IL-18 and Arrests CKD. J Am Soc Nephrol 28(5):1437-1449. https://doi.org/10.1681/ASN.2016070761

95. Szeto HH, Liu S, Soong Y et al (2011) Mitochondria-targeted peptide accelerates ATP recovery and reduces ischemic kidney injury. J Am Soc Nephrol 22(6):1041-1052. https://doi.org/10. 1681/ASN.2010080808

96. Alam NM, Mills WC, Wong AA, Douglas RM, Szeto HH, Prusky GT (2015) A mitochondrial therapeutic reverses visual decline in mouse models of diabetes. Dis Model Mech 8(7):701-710. https:// doi.org/10.1242/dmm.020248

97. Yin X, Manczak M, Reddy PH (2016) Mitochondria-targeted molecules MitoQ and SS31 reduce mutant huntingtin-induced mitochondrial toxicity and synaptic damage in Huntington's disease. Hum Mol Genet 25(9):1739-1753. https://doi.org/10.1093/ $\mathrm{hmg} / \mathrm{ddw} 045$

98. Liu JJ, Green P, John Mann J, Rapoport SI, Sublette ME (2015) Pathways of polyunsaturated fatty acid utilization: implications for brain function in neuropsychiatric health and disease. Brain Res 1597:220-246. https://doi.org/10.1016/j.brainres.2014.11.059

99. Li MY, Wang YY, Cao R et al (2015) Dietary fish oil inhibits mechanical allodynia and thermal hyperalgesia in diabetic rats 
by blocking nuclear factor-kappaB-mediated inflammatory pathways. J Nutr Biochem 26(11):1147-1155. https://doi.org/10.1016/ j.jnutbio.2015.05.005

100. Han E, Yun Y, Kim G et al (2016) Effects of Omega-3 Fatty Acid Supplementation on Diabetic Nephropathy Progression in Patients with Diabetes and Hypertriglyceridemia. PLoS One 11(5): e0154683. https://doi.org/10.1371/journal.pone.0154683

101. Lewis EJH, Perkins BA, Lovblom LE, Bazinet RP, Wolever TMS, Bril V (2017) Effect of omega-3 supplementation on neuropathy in type 1 diabetes: A 12-month pilot trial. Neurology 88(24): 2294-2301. https://doi.org/10.1212/WNL.0000000000004033

102. Sala-Vila A, Diaz-Lopez A, Valls-Pedret C et al (2016) Dietary Marine omega-3 Fatty Acids and Incident Sight-Threatening Retinopathy in Middle-Aged and Older Individuals With Type 2 Diabetes: Prospective Investigation From the PREDIMED Trial. JAMA Ophthalmol 134(10):1142-1149. https://doi.org/10.1001/ jamaophthalmol.2016.2906

103. Herman-Edelstein M, Weinstein T, Levi M (2018) Bile acid receptors and the kidney. Curr Opin Nephrol Hypertens 27(1):56-62. https://doi.org/10.1097/MNH.0000000000000374

104. Wang XX, Wang D, Luo Y et al (2018) FXR/TGR5 Dual Agonist Prevents Progression of Nephropathy in Diabetes and Obesity. J Am Soc Nephrol 29(1):118-137. https://doi.org/10.1681/ASN. 2017020222

105. Hammer SS, Beli E, Kady N et al (2017) The Mechanism of Diabetic Retinopathy Pathogenesis Unifying Key Lipid Regulators, Sirtuin 1 and Liver X Receptor. EBioMedicine 22: 181-190. https://doi.org/10.1016/j.ebiom.2017.07.008

106. Cermenati G, Abbiati F, Cermenati S et al (2012) Diabetesinduced myelin abnormalities are associated with an altered lipid pattern: protective effects of LXR activation. J Lipid Res 53(2): 300-310. https://doi.org/10.1194/jlr.M021188

107. Gavini CK, Bookout AL, Bonomo R, Gautron L, Lee S, MansuyAubert V (2018) Liver X Receptors Protect Dorsal Root Ganglia from Obesity-Induced Endoplasmic Reticulum Stress and Mechanical Allodynia. Cell Rep 25(2):271-277 e274. https:// doi.org/10.1016/j.celrep.2018.09.046
108. Dutta T, Chai HS, Ward LE et al (2012) Concordance of changes in metabolic pathways based on plasma metabolomics and skeletal muscle transcriptomics in type 1 diabetes. Diabetes 61(5):1004 1016. https://doi.org/10.2337/db11-0874

109. Fort PE, Losiewicz MK, Reiter CE et al (2011) Differential roles of hyperglycemia and hypoinsulinemia in diabetes induced retinal cell death: evidence for retinal insulin resistance. PLoS One 6(10): e26498. https://doi.org/10.1371/journal.pone.0026498

110. Hinder LM, Murdock BJ, Park M et al (2018) Transcriptional networks of progressive diabetic peripheral neuropathy in the $\mathrm{db} /$ $\mathrm{db}$ mouse model of type 2 diabetes: An inflammatory story. Exp Neurol 305:33-43. https://doi.org/10.1016/j.expneurol.2018.03. 011

111. Hinder LM, Park M, Rumora AE et al (2017) Comparative RNASeq transcriptome analyses reveal distinct metabolic pathways in diabetic nerve and kidney disease. J Cell Mol Med 21(9):2140 2152. https://doi.org/10.1111/jcmm.13136

112. Crosby-Nwaobi R, Chatziralli I, Sergentanis T, Dew T, Forbes A, Sivaprasad S (2015) Cross Talk between Lipid Metabolism and Inflammatory Markers in Patients with Diabetic Retinopathy. J Diabetes Res 2015:191382

113. Herder C, Lankisch M, Ziegler D et al (2009) Subclinical inflammation and diabetic polyneuropathy: MONICA/KORA Survey F3 (Augsburg, Germany). Diabetes Care 32(4):680-682. https://doi. org/10.2337/dc08-2011

114. Trevisan R, Nosadini R, Fioretto P et al (1987) Metabolic control of kidney hemodynamics in normal and insulin-dependent diabetic subjects. Effects of acetoacetic, lactic, and acetic acids. Diabetes 36(9):1073-1081. https://doi.org/10.2337/diab.36.9.1073

115. Gowda K, Zinnanti WJ, LaNoue KF (2011) The influence of diabetes on glutamate metabolism in retinas. J Neurochem 117(2): 309-320. https://doi.org/10.1111/j.1471-4159.2011.07206.x

Publisher's note Springer Nature remains neutral with regard to jurisdictional claims in published maps and institutional affiliations. 